\title{
Neuroadaptive control of information-poor servomechanisms with smooth and nonsmooth uncertainties
}

\author{
Guichao Yang ${ }^{1}$ (D) Hua Wang ${ }^{1}$ (D)
}

Received: 10 July 2021 / Accepted: 26 November 2021 / Published online: 11 February 2022

(c) The Author(s) 2022

\begin{abstract}
For motion control of uncertain servomechanisms, nonlinear dynamics including smooth and nonsmooth types, external disturbances, signal measurement noises, asymmetric input saturation, and so on seriously hinder the further development of high-performance closed-loop control algorithms. However, already existing control strategies cannot address the abovementioned issues at the same time. It greatly increases the difficulty of controller design especially when some states are not measurable. Inspired by above motivations, this paper exploits neural networks to deal with nonlinear dynamics including discontinuous types, and combines extended state observers to estimate disturbances and unmeasurable states for uncertain nonlinear servomechanisms. Meanwhile, the desired-command-based model compensation approach is integrated into the controller design. It is worth noting that the neural network weights are updated by the combination of the estimation error and tracking error to acquire better approximation accuracy. According to above technologies, a novel extended-state-observerbased neural network adaptive motion control algorithm will be synthesized. The bounded stability of the whole closed-loop system is proved strictly. In addition, the comparisons of the application results on an electro-hydraulic servo system verify the availability and superiority of the developed control algorithm.
\end{abstract}

Keywords Servomechanism $\cdot$ Input saturation $\cdot$ Motion control $\cdot$ Neural network $\cdot$ Extended state observer

\section{Introduction}

Servo mechanical systems, such as servo motor systems [1-4], hydraulic servo systems [5-8], and aerocrafts [9, 10], play an important role in industry and engineering applications, including processing, manufacturing, production, and so on. And they are often affected by unknown nonlinear dynamics, including continuous and discontinuous types, external disturbances, signal measurement noises, input saturation, and other factors. In addition, some signals in the system cannot be measured, because they are not equipped with corresponding sensors. Above-mentioned factors will seriously affect the motion accuracy of servo mechanical systems, leading it difficult to synthesize high-performance closed-loop controllers.

During the past few decades, the motion control for uncertain nonlinear servo systems has mainly revolved around

Hua Wang

wanghua@njtech.edu.cn

1 School of Mechanical and Power Engineering, Nanjing Tech University, Nanjing 211816, China overcoming the modeling uncertainties therein. It is worth noting that a large number of leading control algorithms have been presented along with the development of control theory. Examples like adaptive robust control (ARC) [11], integral robust control (IRC) [12, 13], sliding mode control (SMC) $[14,15]$, disturbance-observer-based control [1, 2, 9, 16-18], neural network (NN)-based control [19-24], and so on. In [25], the authors have presented an integral retarded controller for velocity control of a direct current motor servo system. However, only a part of modeling uncertainties has been dealt with. In [26], for a servo motor actuated gantry system, the authors have developed a friction-compensationbased adaptive SMC control algorithm which utilizes the adaptive control technique to handle uncertain parameters that lie linearly in the system and employs the SMC to deal with external disturbances. However, the final control input contains a discontinuous term, which may cause severe chattering for the actual system. To handle large disturbances existing in a pneumatic servo system, Yuanqing Xia et al. have employed an extended state observer (ESO) to acquire the estimate of lumped nonlinearities and thus compensate for the total disturbances feedforwardly [27]. Furthermore, 
Daehee Won et al. have developed an output feedback integral sliding mode controller by incorporating a high gain observer to acquire the estimate of the unmeasured velocity as well as load pressure [16]. However, unknown nonlinear dynamics have not been considered in [16]. By taking advantage of the good approximation performance of NN for unknown functions, the authors in [7] and [23] have synthesized an adaptive NN (ANN) control algorithm to treat largely uncertain nonlinear dynamics existing in hydraulic cylinder actuated active suspension systems. Furthermore, by exploiting NNs to approximate unknown nonlinear functions and dead zone, the authors in [24] have proposed a ANN control strategy for a family of servo mechanisms. To simultaneously cope with unknown nonlinear dynamic uncertainties and exogenous disturbances, some control strategies which combine neural network with robust control methods have been proposed. In [21], a NN-based ARC control algorithm has been synthesized for a linear motor driven stage to obtain high-accuracy tracking effect and reject external disturbances. In [28], the authors have presented a recurrent NN-based SMC control structure for nonlinear systems to compensate for unknown nonlinear dynamics and suppress external disturbances. Moreover, a NN-based IRC control algorithm has been proposed by Jing $\mathrm{Na}$ et al. for uncertain servomechanisms. Nevertheless, the control strategies mentioned above exist certain conservatism when the considered system is faced with large external disturbances. Furthermore, influenced by external disturbances, the actual control input added to the system may be saturated due to the limitation of electrical hardware. Some control algorithms with saturation compensation [11, 15, 29-31] have been developed for this working condition.

After careful analysis of the existing studies, it is known that scholars mainly deal with the continuous nonlinear dynamic uncertainties via $\mathrm{NN}$, but pay little attention to the existence of discontinuous nonlinear dynamics in the system. As for servomechanisms, there prominently exist various discontinuous nonlinear dynamics, such as discontinuous nonlinear friction, discontinuous hydraulic fluid leakage, and so on. In addition, for feedback control, the measurement values of state feedback information are often needed, but they are usually not measurable for actual systems due to economic, structural, and other factors. As for speed feedback and acceleration feedback in the actual servo mechanical systems, these information are often not obtained by adding corresponding sensors, but by position information via filters. However, the dynamics of these filters are often not included in the closed-loop stability proof of the system, resulting in great conservatism in implementing the final controller. In addition, using the measured value of the signals directly when designing the controller causes a large amount of noises to be mixed, which may lead to unsatisfactory control effects. It is worth noting that most of the existing controllers still use the traditional backstepping method, which will lead to the issue of "explosion of complexity (EC)". By analyzing and summarizing the above studies, it can be found that unknown nonlinear dynamics in the system, especially the types of discontinuities, external interference, measurement noises, and input saturation, and so on, cannot simultaneously be handled under the condition that only some state signals can be measured. And it will also be affected by EC when designing the controller. The above factors bring great challenges to the design of the controller.

Stimulated by above analysis, we will propose a novel control algorithm to deal with the issues. Specially, the proposed control algorithm will employ NN adaptive control [32] to simultaneously estimate the continuous and discontinuous nonlinear dynamics that present in the system. And ESOs [33] will be exploited to estimate unmeasured states, mismatched, and matched exogenous disturbances in the system. By incorporating the desired-command-based technology into NN adaptive control, observer and controller to minimize the influence of signal measurement noises. More importantly, the effect of EC will be eliminated via the command-filtered backstepping technique. And the saturation compensation technology will be utilized to deal with the input saturation effect suffered by the system.

In summary, the proposed control algorithm has the following innovations:

- The proposed control algorithm can simultaneously handle smooth and nonsmooth nonlinear dynamics, matched and mismatched exogenous disturbances, measurement signal noises, and input saturation.

- The better neural network adaptive performance can be acquired, since the weight adaption laws are driven by the composite errors comprised of state prediction errors and system tracking errors.

- The proposed control algorithm can be adapted in the working condition with unmeasurable information, which means that it has strong adaptability to working conditions.

- The integrated control algorithm can not only eliminate the effects of "explosion of complexity", but also avoid the influence of filtering error.

For simplification, some notations are presented as follows:

\begin{tabular}{ll}
\hline$i$ & $1,2,3$ \\
$j$ & 2,3 \\
$b$ & 1,2 \\
$\hat{\mathbf{0}}$ & the estimate of $\bullet$ \\
$\tilde{\boldsymbol{\bullet}}=\boldsymbol{\bullet}-\hat{\bullet}$ & the estimation error of $\bullet$ \\
\hline
\end{tabular}




\section{Problem statement}

A family of third-order uncertain nonlinear servomechanisms in the following state-space form is considered as

$\dot{\chi}_{1}=\chi_{2}$

$\dot{\chi}_{2}=\chi_{3}+\xi_{2}\left(\bar{\chi}_{2}\right)+\varepsilon_{2}(t)$

$\dot{\chi}_{3}=u(v)+\xi_{3}\left(\bar{\chi}_{3}\right)+\psi_{3}\left(\chi_{3}\right)+\varepsilon_{3}(t)$

$y_{o}=\chi_{1}$.

Here, $\chi_{i}$ means the system state variable; $\xi_{j}\left(\bar{\chi}_{j}\right)$ denotes the unknown nonlinear dynamic with respect to $\bar{\chi}_{j}=\left[\chi_{1}\right.$, $\left.\ldots, \chi_{j}\right]^{\mathrm{T}} ; \psi_{3}\left(\chi_{3}\right)$ means the unknown nonlinear dynamic with respect to $\chi_{3} ; \varepsilon_{j}(t)$ represents the lumped time-varying external disturbance; $y_{o}$ stands for the system output; and the input saturation nonlinearity $u(v)$ can be described by

$u(v)=\operatorname{sat}(v)= \begin{cases}\bar{u}, & v \geq \bar{u} \\ v, & \underline{u}<v<\bar{u}, \\ \underline{u}, & v \leq \underline{u}\end{cases}$

where $v$ indicates the control input; moreover, $\bar{u}>0$ and $\underline{u}$ $<0$ denote known bounds for $u(v)$.

Control Goal: To construct a bounded input $v$ with only partial state measurements, so that the $y_{o}=\chi_{1}$ can follow any desired command $\chi_{1 d}(t) \in \Sigma^{1}$ closely in spite of various modeling uncertainties and input saturation nonlinearity.

Before synthesizing the control scheme to acquire the above-mentioned control objective, we firstly make the following several assumptions.

Assumption 1 The desired command $\chi_{1 d}(t)$ belongs to $C^{2}$. The system signals $\chi_{1}$ and $\chi_{3}$ are measurable, while the system signal $\chi_{2}$ is immeasurable. The unknown nonlinear function $\xi_{j}\left(\bar{\chi}_{j}\right)$ belongs to $C^{1}$. Specially, the unknown nonlinear function $\psi_{3}\left(\chi_{3}\right)$ is bounded and continuous except at $\chi_{3}=c_{c}$ where it exhibits a finite jump and is always continuous from the right, in which $c_{c}$ means a constant.

Assumption 2 There exist some positive constants $\varepsilon_{2 m}$ and $\varepsilon_{3 m}$, such that exogenous disturbances $\varepsilon_{2}(t)$ and $\varepsilon_{3}(t)$ fulfill

$\left|\varepsilon_{2}(t)\right| \leq \varepsilon_{2 m},\left|\varepsilon_{3}(t)\right| \leq \varepsilon_{3 m}$.

Lemma 1 Denote a command filter as follows [31]:

$\dot{x}_{1}=w_{c} x_{2}$

$\dot{x}_{2}=-2 w_{f} w_{c} x_{2}-w_{c}\left(x_{1}-x_{r}\right)$.

If the input variable $x_{r}$ fulfills $\left|\dot{x}_{r}(0)\right| \leq x_{r 1}$ and $\left|\ddot{x}_{r}(0)\right| \leq x_{r 2}$ for any time $t \geq 0$ with $x_{r 1}$ and $x_{r 2}$ being some positive constants, and meanwhile, $x_{1}(0)=x_{r}(0), x_{2}(0)=0$, hence there exist positive constants $\omega_{c}, \varepsilon_{c}$, and $0<\omega_{f}<1$, so that $\left|x_{1}-x_{r}\right| \leq \varepsilon_{c}$.

Remark 1 For a large number of practical applications, typical examples like hydraulic/motor servo systems $[1,2,6,18$, 34], they can always be modeled or transformed as (1). Furthermore, some discontinuous nonlinear dynamics, such as discontinuous friction and discontinuous leakage, which are not ignored have yet been considered in (1). In addition, it can be found from (1) that mismatched modeling uncertainties including $\xi_{2}\left(\bar{\chi}_{2}\right)$ and $\varepsilon_{2}(t)$, matched modeling uncertainties including $\xi_{3}\left(\bar{\chi}_{3}\right)$ and $\varepsilon_{3}(t)$, and input saturation nonlinearity are simultaneously taken into account.

\section{Command-filter-based neural network controller with partial state feedback}

\section{Neural network approximation}

According to the powerful universal approximation characteristic of $\mathrm{NN}$, for any $C^{1}$ nonlinear function $F_{1}(x)$, there exist ideal weights $W_{*} \in \mathbb{R}^{L}$ with $L$ being the number of RBFNN nodes, so that [32]

$F_{1}(x)=W_{*}^{T} \eta_{*}(x)+\sigma(x)$,

where $x$ means the input vector, $\sigma(x)$ indicates the approximation error, and $\eta_{*}(x)=\left[\eta_{* 1}(x), \eta_{* 2}(x), \ldots, \eta_{* L}(x)\right]^{T}$ with the function $\eta_{* l}(x), l=1,2, \ldots, L$, usually being chosen as the Gaussian RBF [32]

$\eta_{* l}(x)=e^{-\left\|x-c_{l}\right\|^{2} / 2 w_{l}^{2}}$,

in which $c_{l}$ and $w_{l}$ are center vectors and widths of the RBF, respectively.

Noting (5), the function $F_{1}(x)$ is able to be approximated by

$\hat{F}_{1}(x)=\hat{W}_{*}^{T} \eta_{*}(x)$.

Furthermore, for any continuously nonlinear function $F_{2}(x)$ except at $x=c_{c}$ where it exhibits a jump and is also continuous from the right, there exist ideal weights $G * \in \mathbb{R}^{M}$ with $M$ being the number of $\mathrm{NN}$ nodes, so that

$F_{2}(x)=G_{*}^{T} \psi_{*}(x)+\mu_{c}(x)$,

where $\mu_{c}(x)$ indicates the approximation error; and $\psi_{*}(x)=$ $\left[\psi_{* 1}(x), \psi_{* 2}(x), \ldots, \psi_{* M}(x)\right]^{T}$ with the sigmoid jump function $\psi_{* m}(x), m=0,1, \ldots, M$, usually being chosen as follows [35]:

$\psi_{* m}(x)=\left\{\begin{array}{ll}\left(1-e^{-x}\right)^{m} & \text { if } x \geq 0 \\ 0 & \text { otherwise }\end{array}\right.$. 
Based on (8), $F_{2}(x)$ can be estimated by

$\hat{F}_{2}(x)=\hat{G}_{*}^{T} \psi_{*}(x)$

\section{NN-based ESOs design}

Noting the considered system in (1), we can reorganize the expression $\xi_{3}\left(\bar{\chi}_{3}\right)$ as

$\xi_{3}\left(\bar{\chi}_{3}\right)=\varphi_{3}\left(\bar{\chi}_{2}, \dot{\chi}_{2}\right)+\varepsilon_{4}(t)$,

where $\varphi_{3}\left(\bar{\chi}_{2}, \dot{\chi}_{2}\right)$ indicates a nonlinear function with respect to $\bar{\chi}_{2}$ and $\dot{\chi}_{2}$, and $\varepsilon_{4}(t)$ represents the lumped time-varying external disturbance.

Based on (1) and (11), the nonlinear system (1) can be reorganized as

$\dot{\chi}_{1}=\chi_{2}$

$\dot{\chi}_{2}=\chi_{3}+\xi_{2}\left(\bar{\chi}_{2 d}\right)+\tilde{N}_{2}+\varepsilon_{2}(t)$

$\dot{\chi}_{3}=u(v)+\varphi_{3}\left(\bar{\chi}_{3 d}\right)+\tilde{N}_{3}+\varepsilon_{4}(t)+\psi_{3}\left(\chi_{3}\right)+\varepsilon_{3}(t)$,

where $\bar{\chi}_{2 d}=\left[\chi_{1 d}, \dot{\chi}_{1 d}\right]^{T}$ and $\bar{\chi}_{3 d}=\left[\chi_{1 d}, \dot{\chi}_{1 d}, \ddot{\chi}_{1 d}\right]^{T}$ are vectors with respect to desired commands. In addition, $\tilde{N}_{2}$ and $\tilde{N}_{3}$ are expressed as

$\tilde{N}_{2} \triangleq \xi_{2}\left(\bar{\chi}_{2}\right)-\xi_{2}\left(\bar{\chi}_{2 d}\right), \tilde{N}_{3} \triangleq \varphi_{3}\left(\bar{\chi}_{2}, \dot{\chi}_{2}\right)-\varphi_{3}\left(\bar{\chi}_{3 d}\right)$.

Depending on (5), (8), (12), and (13), the system (1) can be equivalent to the form as below

$$
\begin{aligned}
\dot{\chi}_{1}= & \chi_{2} \\
\dot{\chi}_{2}= & \chi_{3}+W_{2}^{T} \eta_{2}\left(\bar{\chi}_{2 d}\right)+\sigma_{2}\left(\bar{\chi}_{2 d}\right)+\tilde{N}_{2}+\varepsilon_{2}(t) \\
\dot{\chi}_{3}= & u(v)+W_{3}^{T} \eta_{3}\left(\bar{\chi}_{3 d}\right)+\sigma_{3}\left(\bar{\chi}_{3 d}\right)+\tilde{N}_{3}+\varepsilon_{4}(t) \\
& +G^{T} \psi_{p}\left(\chi_{3}\right)+\mu\left(\chi_{3}\right)+\varepsilon_{3}(t),
\end{aligned}
$$

where $\eta_{2}\left(\bar{\chi}_{2 d}\right)$ and $\eta_{3}\left(\bar{\chi}_{3 d}\right)$ are Gaussian RBFs whose expressions are similar to $(6) ; \psi_{p}\left(\chi_{3}\right)$ indicates the sigmoid jump function whose expression is similar to (9); in addition, $\sigma_{2}\left(\bar{\chi}_{2 d}\right), \sigma_{3}\left(\bar{\chi}_{3 d}\right)$ and $\mu\left(\chi_{3}\right)$ represent different approximation errors.

First, let us introduce two variables $\chi_{D 2}(t)=\sigma_{2}\left(\bar{\chi}_{2 d}\right)+$ $\varepsilon_{2}(t)$ and $\chi_{D 3}(t)=\sigma_{3}\left(\bar{\chi}_{3 d}\right)+\varepsilon_{3}(t)+\varepsilon_{4}(t)$ as extended states; thus, the state-space expression (14) can be converted into the following form:

$$
\begin{aligned}
& \left\{\begin{array}{l}
\dot{\chi}_{1}=\chi_{2} \\
\dot{\chi}_{2}=\chi_{3}+W_{2}^{T} \eta_{2}\left(\bar{\chi}_{2 d}\right)+\chi_{D 2}+\tilde{N}_{2} \\
\dot{\chi}_{D 2}=\alpha_{1}(t)
\end{array}\right. \\
& \left\{\begin{array}{c}
\dot{\chi}_{3}=u(v)+W_{3}^{T} \eta_{3}\left(\bar{\chi}_{3 d}\right)+\tilde{N}_{3} \\
+G^{T} \psi_{p}\left(\chi_{3}\right)+\mu\left(\chi_{3}\right)+\chi_{D 3} . \\
\dot{\chi}_{D 3}=\alpha_{2}(t)
\end{array}\right.
\end{aligned}
$$

Thus, the NN-based ESOs for (15) can be synthesized as

$$
\begin{aligned}
& \left\{\begin{array}{c}
\dot{\hat{\chi}}_{1}=\hat{\chi}_{2}+\gamma_{1} \omega_{o 1}\left(\chi_{1}-\hat{\chi}_{1}\right) \\
\dot{\hat{\chi}}_{2}=\chi_{3}+\hat{W}_{2}^{T} \eta_{2}\left(\bar{\chi}_{2 d}\right)+\hat{\chi}_{D 2}+\gamma_{2} \omega_{o 1}^{2}\left(\chi_{1}-\hat{\chi}_{1}\right) \\
\dot{\hat{\chi}}_{D 2}=\gamma_{3} \omega_{o 1}^{3}\left(\chi_{1}-\hat{\chi}_{1}\right)
\end{array}\right. \\
& \left\{\begin{array}{c}
\dot{\hat{\chi}}_{3}=u(v)+\hat{W}_{3}^{T} \eta_{3}\left(\bar{\chi}_{3 d}\right)+\hat{G}^{T} \psi_{p}\left(\chi_{3}\right) \\
\quad+\hat{\chi}_{D 3}+r_{1} \omega_{o 2}\left(\chi_{3}-\hat{\chi}_{3}\right) \\
\dot{\hat{\chi}}_{D 3}=r_{2} \omega_{o 2}^{2}\left(\chi_{3}-\hat{\chi}_{3}\right)
\end{array}\right.
\end{aligned}
$$

where the adjustable gains $\omega_{o 1}>0$ and $\omega_{o 2}>0$ can be seen as the bandwidths of the designed observers; design parameters $\gamma_{i}$ and $r_{b}$ need to be chosen to guarantee that the characteristic polynomials $\gamma_{1} s^{2}+\gamma_{2} s+\gamma_{3}$ and $r_{1} s+r_{2}$ of the following matrices $C_{o}$ and $R_{o}$ are Hurwitz, respectively. It is worth noted from [36] that $\gamma_{i}$ and $r_{b}$ can be parameterized as $\gamma_{1}=$ $3, \gamma_{2}=3, \gamma_{3}=1$ and $r_{1}=2, r_{2}=1$, respectively.

Upon (15) and (16), the estimation error dynamics of the constructed observers are arranged as

$$
\begin{aligned}
& \left\{\begin{array}{l}
\dot{\tilde{\chi}}_{1}=-\gamma_{1} \omega_{o 1} \tilde{\chi}_{1}+\tilde{\chi}_{2} \\
\dot{\tilde{\chi}}_{2}=-\gamma_{2} \omega_{o 1}^{2} \tilde{\chi}_{1}+\tilde{\chi}_{D 2}+\tilde{W}_{2}^{T} \eta_{2}\left(\bar{\chi}_{2 d}\right)+\tilde{N}_{2} \\
\dot{\tilde{\chi}}_{D 2}=-\gamma_{3} \omega_{o 1}^{3} \tilde{\chi}_{1}+\alpha_{1}(t)
\end{array}\right. \\
& \left\{\begin{array}{c}
\dot{\tilde{\chi}}_{3}=-r_{1} \omega_{o 2} \tilde{\chi}_{3}+\tilde{\chi}_{D 3}+\tilde{W}_{3}^{T} \eta_{3}\left(\bar{\chi}_{3 d}\right) \\
+\tilde{N}_{3}+\tilde{G}^{T} \psi_{p}\left(\chi_{3}\right)+\mu\left(\chi_{3}\right) \\
\dot{\tilde{\chi}}_{D 3}=-r_{2} \omega_{o 2}^{2} \tilde{\chi}_{3}+\alpha_{2}(t)
\end{array}\right.
\end{aligned}
$$

For facilitating the subsequent theoretical analysis, a set of state vectors $\zeta=\left[\zeta_{1}, \zeta_{2}, \zeta_{3}\right]^{T}=\left[\tilde{\chi}_{1}, \tilde{\chi}_{2} / \omega_{o 1}, \tilde{\chi}_{D 2} / \omega_{o 1}^{2}\right]^{T}$ and $\delta=\left[\delta_{1}, \delta_{2}\right]^{T}=\left[\tilde{\chi}_{3}, \tilde{\chi}_{D 3} / \omega_{o 2}\right]^{T}$ are introduced. Hence, (17) can be rearranged as

$$
\begin{aligned}
\dot{\zeta} & =\omega_{o 1} C_{o} \zeta+H_{1} \frac{\tilde{W}_{2}^{T} \eta_{2}\left(\bar{\chi}_{2 d}\right)+\tilde{N}_{2}}{\omega_{o 1}}+H_{2} \frac{\alpha_{1}(t)}{\omega_{o 1}^{2}} \\
\dot{\delta} & =\omega_{o 2} R_{o} \delta+S_{1}\left[\tilde{W}_{3}^{T} \eta_{3}\left(\bar{\chi}_{3 d}\right)+\tilde{N}_{3}\right] \\
& +S_{1}\left[\tilde{G}^{T} \psi_{p}\left(\chi_{3}\right)+\mu\left(\chi_{3}\right)\right]+S_{2} \frac{\alpha_{2}(t)}{\omega_{o 2}}
\end{aligned}
$$

in which

$$
\begin{aligned}
& C_{o}=\left[\begin{array}{lll}
-\gamma_{1} & 1 & 0 \\
-\gamma_{2} & 0 & 1 \\
-\gamma_{3} & 0 & 0
\end{array}\right], \quad H_{1}=\left[\begin{array}{l}
0 \\
1 \\
0
\end{array}\right], \quad H_{2}=\left[\begin{array}{l}
0 \\
0 \\
1
\end{array}\right] \\
& R_{o}=\left[\begin{array}{ll}
-r_{1} & 1 \\
-r_{2} & 0
\end{array}\right], \quad S_{1}=\left[\begin{array}{l}
1 \\
0
\end{array}\right], \quad S_{2}=\left[\begin{array}{l}
0 \\
1
\end{array}\right] .
\end{aligned}
$$

As the matrices $C_{o}$ and $R_{o}$ are Hurwitz, there must exist positive definite matrices $E_{o}$ and $Y_{o}$, such that $C_{o}^{T} E_{o}+E_{o} C_{o}$ $=-I$ and $R_{o}^{T} Y_{o}+Y_{o} R_{o}=-I$ always hold [37]. Furthermore, 
according to aforementioned parameterized values $\gamma_{i}$ and $r_{b}$, $E_{o}$ and $Y_{o}$ can be, respectively, calculated as

$$
E_{O}=\left[\begin{array}{ccc}
1 & -\frac{1}{2} & -1 \\
-\frac{1}{2} & 1 & -\frac{1}{2} \\
-1 & -\frac{1}{2} & 4
\end{array}\right], Y_{O}=\left[\begin{array}{cc}
\frac{1}{2} & -\frac{1}{2} \\
-\frac{1}{2} & \frac{3}{2}
\end{array}\right] .
$$

Furthermore, the theoretical analysis for the stability of the constructed NN-based ESOs which is included in that of the whole closed-loop system will be provided in Appendix A.

\section{Command filtered model compensation controller with partial state feedback}

To synthesize the subsequent control scheme, we define a set of tracking error variables $z_{i}$ and error compensation signals $e_{i}$ as follows:

$$
\begin{array}{r}
z_{1}=\chi_{1}-\chi_{1 d}, e_{1}=z_{1}-\phi_{1} \\
z_{2}=\chi_{2}-v_{1}, e_{2}=z_{2}-\phi_{2} \\
z_{3}=\chi_{3}-v_{2, c}, e_{3}=z_{3}-\phi_{3},
\end{array}
$$

where $v_{1}$ and $v_{2}$ indicate virtual control functions; $v_{2, c}$ denotes the filtered signal of $v_{2}$ via the presented command filter in (4). In addition, a set of compensating signals $\phi_{i}$ is introduced as follows:

$$
\begin{aligned}
& \dot{\phi}_{1}=-k_{1} \phi_{1}+\phi_{2} \\
& \dot{\phi}_{2}=-k_{2} \phi_{2}+\phi_{3}+\left(v_{2, c}-v_{2}\right) \\
& \dot{\phi}_{3}=-k_{3} \phi_{3}+\Delta u,
\end{aligned}
$$

where $k_{i}$ indicates positive feedback gains and $\Delta u=u(v)-v$.

Step 1:

Upon (1), (11), and (12), the time derivative of $e_{1}$ is produced as

$$
\begin{gathered}
\dot{e}_{1}=e_{2}+\phi_{2}+v_{1}-\dot{\chi}_{1 d}-\left(-k_{1} \phi_{1}+\phi_{2}\right) \\
=e_{2}+v_{1}-\dot{\chi}_{1 d}+k_{1} \phi_{1} .
\end{gathered}
$$

Thus, the virtual control function $v_{1}$ is synthesized as

$v_{1}=-k_{1} z_{1}+\dot{\chi}_{1 d}$.

After substituting (26) into (25), we have

$$
\dot{e}_{1}=-k_{1} e_{1}+e_{2} \text {. }
$$

\section{Step 2:}

Noting (14), (23), and (24), we can get the time derivative of $e_{2}$ as

$$
\dot{e}_{2}=\dot{\chi}_{2}-\dot{v}_{1}-\dot{\phi}_{2}
$$

$$
\begin{aligned}
= & \chi_{3}+W_{2}^{T} \eta_{2}\left(\bar{\chi}_{2 d}\right)+\chi_{D 2}+\tilde{N}_{2} \\
& -\left(-k_{1} \dot{z}_{1}+\ddot{\chi}_{1 d}\right)+k_{2} \phi_{2}-\phi_{3}-\left(v_{2, c}-v_{2}\right) .
\end{aligned}
$$

As

$$
\begin{aligned}
\dot{z}_{1} & =\dot{e}_{1}+\dot{\phi}_{1} \\
& =-k_{1} e_{1}+e_{2}-k_{1} \phi_{1}+\phi_{2} .
\end{aligned}
$$

We can achieve

$$
\begin{aligned}
\dot{e}_{2}= & e_{3}+v_{2}+k_{2} \phi_{2}+W_{2}^{T} \eta_{2}\left(\bar{\chi}_{2 d}\right)+\chi_{D 2} \\
& +\tilde{N}_{2}+k_{1}\left(-k_{1} e_{1}+e_{2}-k_{1} \phi_{1}+\phi_{2}\right)-\ddot{\chi}_{1 d} .
\end{aligned}
$$

Therefore, we can construct the virtual control function $v_{2}$ as

$v_{2}=-k_{2}\left(\hat{\chi}_{2}-v_{1}\right)-\hat{W}_{2}^{T} \eta_{2}\left(\bar{\chi}_{2 d}\right)-\hat{\chi}_{D 2}+\ddot{\chi}_{1 d}-e_{1}$.

After substituting (31) into (30), one has

$$
\begin{aligned}
\dot{e}_{2}= & e_{3}-k_{2} e_{2}-e_{1}+k_{2} \tilde{\chi}_{2}+\tilde{W}_{2}^{T} \eta_{2}\left(\bar{\chi}_{2 d}\right) \\
& +\tilde{\chi}_{D 2}+\tilde{N}_{2}-k_{1}^{2} e_{1}+k_{1} e_{2}-k_{1}^{2} \phi_{1}+k_{1} \phi_{2} .
\end{aligned}
$$

Step 3:

Based on (14), (23), and (24), we are able to acquire the time derivative of $e_{3}$ as

$$
\begin{aligned}
\dot{e}_{3}= & \dot{\chi}_{3}-\dot{v}_{2, c}-\dot{\phi}_{3} \\
= & u(v)+W_{3}^{T} \eta_{3}\left(\bar{\chi}_{3 d}\right)+\tilde{N}_{3}+G^{T} \psi_{p}\left(\chi_{3}\right) \\
& +\mu\left(\chi_{3}\right)+\chi_{D 3}-\dot{v}_{2, c}-\left(-k_{3} \phi_{3}+\Delta u\right) \\
= & v+k_{3} \phi_{3}+W_{3}^{T} \eta_{3}\left(\bar{\chi}_{3 d}\right)+\tilde{N}_{3} \\
& +G^{T} \psi_{p}\left(\chi_{3}\right)+\mu\left(\chi_{3}\right)+\chi_{D 3}-\dot{v}_{2, c} .
\end{aligned}
$$

Noting (33), the resulting control law $v$ can be synthesized by

$$
\begin{aligned}
v= & -k_{3}\left(\chi_{3}-v_{2, c}\right)-\hat{W}_{3}^{T} \eta_{3}\left(\bar{\chi}_{3 d}\right) \\
& -\hat{G}^{T} \psi_{p}\left(\chi_{3}\right)-\hat{\chi}_{D 3}+\dot{v}_{2, c}-e_{2} .
\end{aligned}
$$

After substituting (34) into (33), one gets

$$
\begin{aligned}
\dot{e}_{3}= & -k_{3} e_{3}-e_{2}+\tilde{W}_{3}^{T} \eta_{3}\left(\bar{\chi}_{3 d}\right) \\
& +\tilde{N}_{3}+\tilde{G}^{T} \psi\left(\chi_{3}\right)+\mu\left(\chi_{3}\right)+\tilde{\chi}_{D 3} .
\end{aligned}
$$

The framework of the developed controller is shown in Fig. 1. 


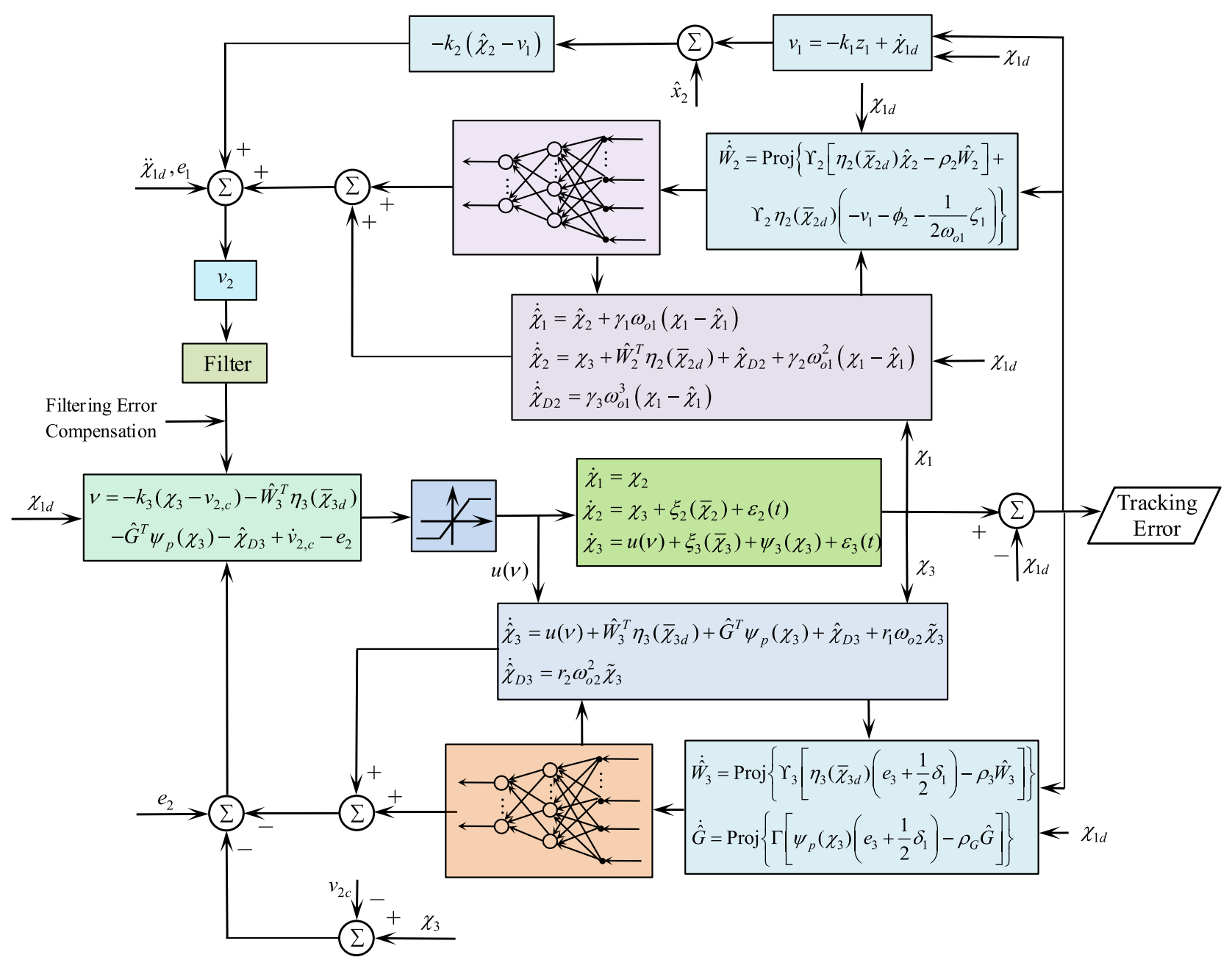

Fig. 1 The framework of the developed control scheme

\section{Main results}

Theorem 1 With the NNs' weights online updated by

$$
\begin{aligned}
& \dot{\hat{W}}_{2}=\operatorname{Proj}\left\{\Upsilon_{2}\left[\eta_{2}\left(\bar{\chi}_{2 d}\right)\left(\hat{\chi}_{2}-v_{1}-\phi_{2}-\frac{1}{2 \omega_{o 1}} \zeta_{1}\right)-\rho_{2} \hat{W}_{2}\right]\right\} \\
& \dot{\hat{W}}_{3}=\operatorname{Proj}\left\{\Upsilon_{3}\left[\eta_{3}\left(\bar{\chi}_{3 d}\right)\left(e_{3}+\frac{1}{2} \delta_{1}\right)-\rho_{3} \hat{W}_{3}\right]\right\} \\
& \dot{\hat{G}}=\operatorname{Proj}\left\{\Gamma\left[\psi_{p}\left(\chi_{3}\right)\left(e_{3}+\frac{1}{2} \delta_{1}\right)-\rho_{G} \hat{G}\right]\right\}
\end{aligned}
$$

in which $\operatorname{Proj}(\bullet)$ indicates a smooth projection operator; $\Upsilon_{2}$, $\Upsilon_{3}$, and $\Gamma$ mean positive definite and symmetric adjustable gain matrices; moreover, $\rho_{2}, \rho_{3}$, and $\rho_{G}$ indicate adjustable positive gains. By choosing design parameters $w_{c}, w_{f}$ for the introduced command filter and other positive gains including $\omega_{o 1}, \omega_{o 2}, k_{i}$ felicitously, thus the integrated control algorithm can make sure that all signals in the closedloop servomechanism (1) with largely unknown mismatched, matched modeling uncertainties and input saturation nonlinearity are bounded.

Proof See Appendix A.

Remark 2 Notably, the developed control strategy can compensate for largely uncertain nonlinear dynamics and external disturbances with only partial state measurements. Specially, discontinuous nonlinear dynamics are also handled. And a command filter is introduced to remove the effect of EC. Moreover, by introducing a series of compensation functions, not only the input saturation effect is compensated, but also the filtering error is eliminated [38, 39]. Furthermore, measurement noises are suppressed by introducing the desired compensation technique.

\section{Application verification}

To check the performance of the above-mentioned control strategy in different aspects, we will carry out numerical sim- 
ulations for a typical verification platform, i.e., a double-rod electric-hydraulic position servomechanism [8]. Moreover, random measurement noises are added.

By introducing a set of state variables $\chi_{1}=y_{L}, \chi_{2}=\dot{y}_{L}$, and $\chi_{3}=A_{J} P_{L} / J$, the nonlinear model of the double-rod hydraulic position servomechanism in [8] can be written as

$$
\begin{aligned}
& \dot{\chi}_{1}=\chi_{2} \\
& \dot{\chi}_{2}=\chi_{3}+\xi_{2}\left(\bar{\chi}_{2}\right)+\varepsilon_{2}(t) \\
& \dot{\chi}_{3}=U(v)+\xi_{3}\left(\bar{\chi}_{3}\right)+\psi_{3}\left(\chi_{3}\right)+\varepsilon_{3}(t),
\end{aligned}
$$

where $U(v)=g_{m} u(v)$ with $g_{m}$ being $g_{m}=4 A_{J} \beta_{o e} k_{t} k_{v} /\left(J V_{t}\right)$. Specially, the definitions of the system variables are presented as follows.

\begin{tabular}{ll}
\hline$J$ & The load mass \\
$y_{L}$ & The displacement of the load \\
$V_{t}$ & The total volume of the hydraulic chambers \\
$A_{J}$ & The active area of the piston rod \\
$P_{s}$ & The oil supply pressure \\
$P_{r}$ & The oil return pressure \\
$P_{L}$ & The load pressure \\
$\beta_{\mathrm{oe}}$ & The effective bulk modulus of oil \\
$k_{v}$ & The gain of the employed servo valve \\
$k_{t}$ & The discharge coefficient \\
\hline
\end{tabular}

It is worth noted from (37) that an additional variable $U(v)$ is employed, which means that the actual control input $v$ can be calculated once $U(v)$ is synthesized out. Moreover, the central physical values of the system parameters for the employed hydraulic servomechanism are collected in Table 1.

To indicate the high-performance tracking effect of the developed algorithm, the following controllers are set for comparison in two working conditions.

(1) C1: This is the integrated control scheme in this paper, i.e., the NN controller with disturbance and nonlinear

Table 1 The central physical values for the hydraulic servomechanism

\begin{tabular}{|c|c|}
\hline The positive feedback gains & $k_{1}=200, k_{2}=600, k_{3}=100$ \\
\hline The gains for the ESOs & $\omega_{o 1}=200, \omega_{o 2}=1500$ \\
\hline $\begin{array}{l}\text { The design parameters for the } \\
\text { NNs }\end{array}$ & $\begin{array}{l}\Upsilon_{2}=500 I_{9 \times 9}, \Upsilon_{3}= \\
1000 I_{11 \times 11}, \Gamma=10 I_{16 \times 16}, \rho_{2} \\
=0.1, \rho_{3}=0.1, \rho_{G}=1 ; \text { the } \\
\text { center vectors for } \eta_{2}\left(\bar{\chi}_{2 d}\right) \text { and } \\
\eta_{3}\left(\bar{\chi}_{3 d}\right) \text { are evenly spaced in } \\
{[-1.2,1.2] \text { and }[-2,2],} \\
\text { respectively; the widths for } \\
\eta_{2}\left(\bar{\chi}_{2 d}\right) \text { and } \eta_{3}\left(\bar{\chi}_{3 d}\right) \text { are } 1 \\
\text { and 5, respectively }\end{array}$ \\
\hline $\begin{array}{l}\text { The design parameters of the } \\
\text { command filter for } v_{2}\end{array}$ & $\omega_{f}=0.99, \omega_{c}=1500$ \\
\hline $\begin{array}{l}\text { The bounds for } u(v) \text { in working } \\
\text { condition } 1\end{array}$ & $\bar{u}=1.12, \underline{u}=-1.12$ \\
\hline $\begin{array}{l}\text { The bounds for } u(v) \text { in working } \\
\text { condition } 2\end{array}$ & $\bar{u}=2.1, \underline{u}=-2.2$ \\
\hline
\end{tabular}

\begin{tabular}{ll}
\hline Parameters & Values (unit) \\
\hline$J$ & $30(\mathrm{~kg})$ \\
$V_{t}$ & $7.962 \times 10^{-5}\left(\mathrm{~m}^{3}\right)$ \\
$A_{J}$ & $9.0478 \times 10^{-4}\left(\mathrm{~m}^{2}\right)$ \\
$\beta_{o e}$ & $7 \times 10^{8}(\mathrm{~Pa})$ \\
$k_{v}$ & $2.5 \times 10^{-3}(\mathrm{~m} / \mathrm{V})$ \\
$k_{t}$ & $5 \times 10^{-2}\left(\mathrm{~m}^{3} / \mathrm{s}\right)$ \\
$P_{s}$ & $1 \times 10^{7}(\mathrm{~Pa})$ \\
$P_{r}$ & 0 \\
\hline
\end{tabular}

Table 2 The design parameters of the $\mathrm{C} 1$ controller

dynamics compensation via partial state information feedback. To make it clear, the design parameters of this controller are presented in Table 2.

(2) C2: This is the controller which is same as the aforementioned $\mathrm{C} 1$ control scheme but without external disturbance compensation. The objective of setting such a controller is to test the disturbance compensation performance of the developed $\mathrm{C} 1$ controller.

(3) C3: This is the controller which is same as the aforementioned $\mathrm{C} 1$ control algorithm but without NN adaptive and disturbance compensation.

(4) C4: This is the integral robust controller whose control scheme is integrated as follows:

$$
\begin{aligned}
z_{1}= & \chi_{1}-\chi_{1 d}, z_{2}=\chi_{2}-v_{1}, \\
z_{3}= & \chi_{3}-v_{2}, v_{1} \triangleq-k_{1} z_{1}+\dot{\chi}_{1 d}, \\
v_{2}= & \dot{v}_{1}-k_{2} z_{2}-k_{r} z_{2} \\
& -\int_{0}^{t}\left[k_{r} k_{2} z_{2}+\beta_{r 2} \operatorname{sign}\left(z_{2}\right) d \tau,\right. \\
u= & {\left[-k_{3} z_{3}+\dot{v}_{2}-\beta_{r 3} \operatorname{sign}\left(z_{3}\right)\right] / g_{m} . }
\end{aligned}
$$

The gains of this controller are presented as $k_{r}=80, \beta_{r 2}$ $=80$, and $\beta_{r 3}=80$, respectively.

(5) C5: This is the distinguished proportional integral controller with its proportional gain and integral gain being 1500 and 600, respectively.

For fair comparison, all corresponding design parameters of $\mathrm{C} 2, \mathrm{C} 3$, and $\mathrm{C} 4$ controllers are chosen as same as that of the $\mathrm{C} 1$ controller. 

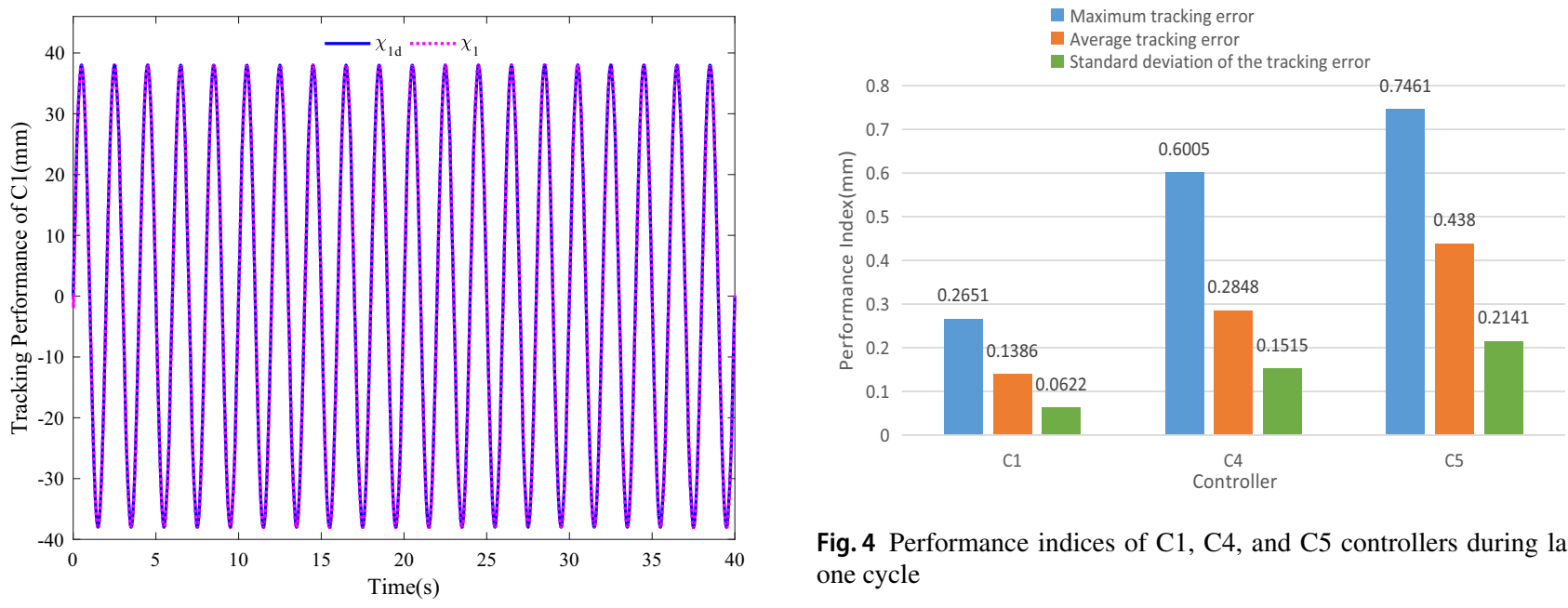

Fig. 4 Performance indices of $\mathrm{C} 1, \mathrm{C} 4$, and C5 controllers during last one cycle

Fig. 2 The tracking performance of $\mathrm{C} 1$
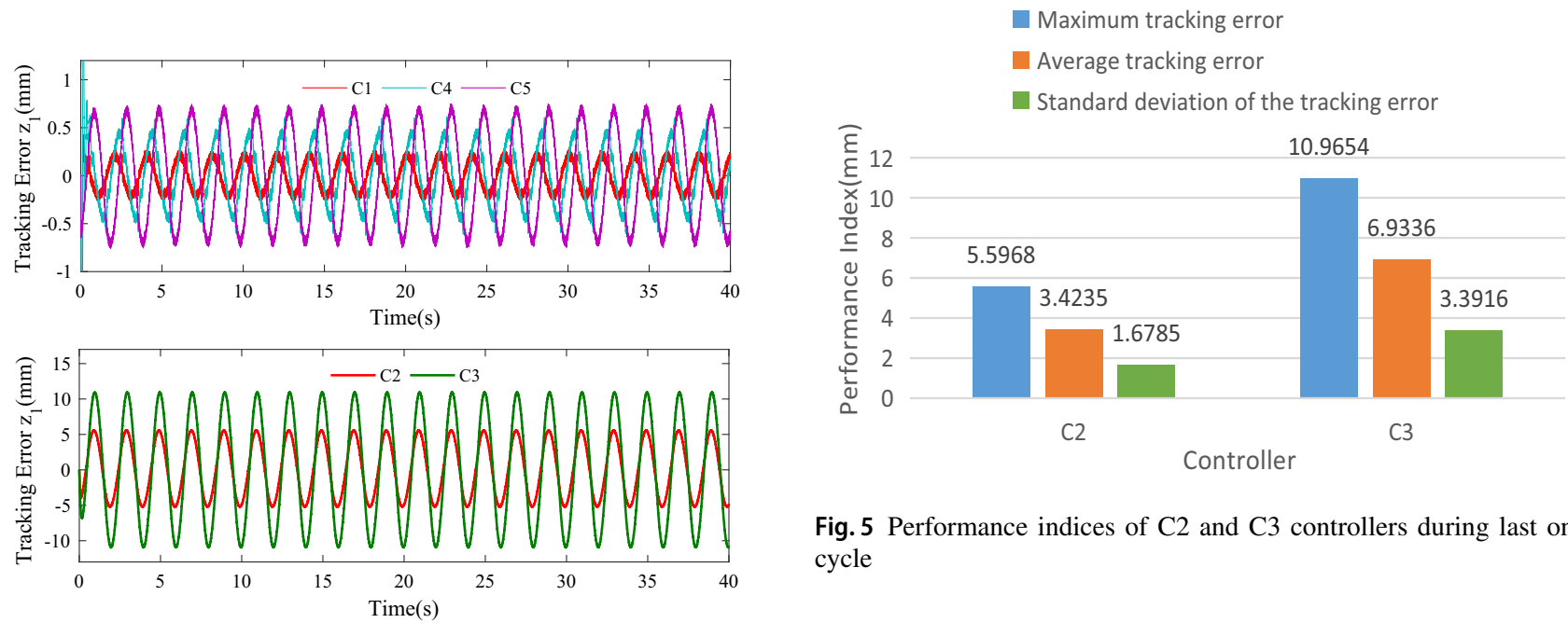

Fig. 5 Performance indices of $\mathrm{C} 2$ and $\mathrm{C} 3$ controllers during last one cycle

Fig. 3 Tracking errors of the contradistinctive controllers

\section{Working condition 1: normal motion}

To indicate the high-performance tracking effects of the synthesized $\mathrm{C} 1$ controller, we employ the desired motion trajectory $\chi_{1 d}(t)=38 \sin (\pi t) \mathrm{mm}$ which is displayed in Fig. 2 . Moreover, we can see from Fig. 2 that the output $\chi_{1}$ under the $\mathrm{C} 1$ controller can track $\chi_{1 d}$ closely. Furthermore, the tracking errors of the employed five controllers are shown in Fig. 3. From them, we can easily find that the synthesized $\mathrm{C} 1$ controller in this paper expresses best tracking performance than the other three controllers in terms of transient and steadystate performance. Moreover, the high tracking performance of $\mathrm{C} 1$ can be indicated via the presented performance indices in Figs. 4 and 5. It is noted that the reason why the $\mathrm{C} 1$ controller exhibits such excellent control performance is due to its model compensations for nonlinear dynamics and distur-

bances. In detail, the availability of the developed adaptive NN-based nonlinear dynamics compensation can be verified by comparing the tracking errors of $\mathrm{C} 2$ and $\mathrm{C} 3$. Furthermore, the validity of the developed ESO-based disturbance compensation performance can be proved by comparing the tracking errors of $\mathrm{C} 1$ and $\mathrm{C} 2$. Specially, the good state estimation performance and disturbance estimation performance from Figs. 6 and 7 illustrate the effectiveness of the developed ESOs. In addition, the estimation performance of nonlinear functions with the $\mathrm{C} 1$ controller in Fig. 8, who is regular and bounded, shows that the developed $\mathrm{NN}$ adaptive control has good function approximation performance. The effectiveness of combining nonlinear dynamics and disturbance compensation can be illustrated by comparing the tracking errors of $\mathrm{C} 1$ and $\mathrm{C} 3$. From Fig. 9, we can make out that the actual control input is always within the preset ranges and presents corresponding rules, even in the case of input saturation. 

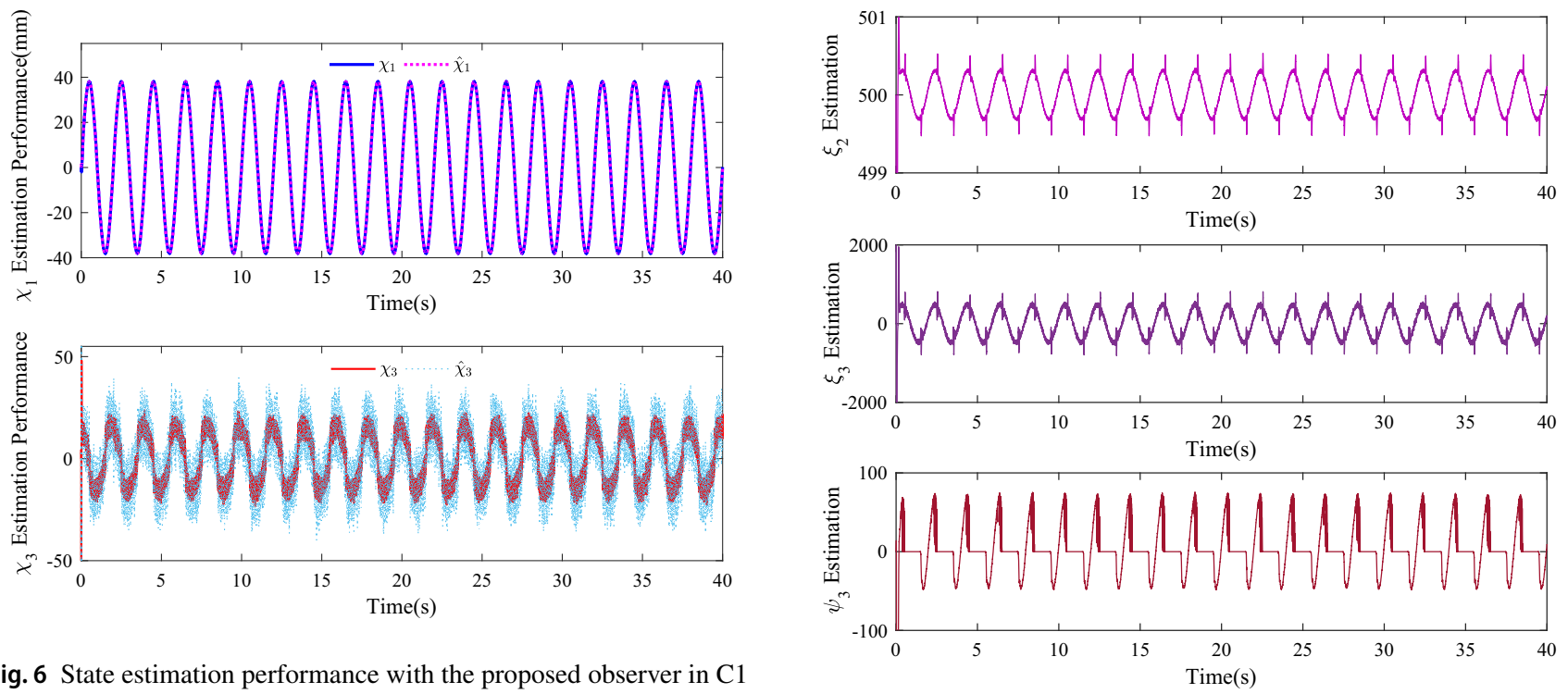

Fig. 6 State estimation performance with the proposed observer in C1
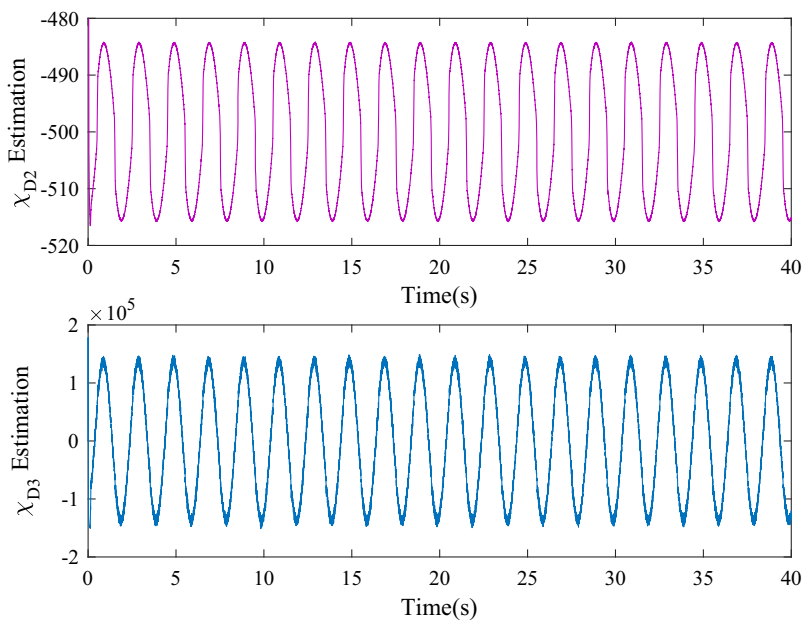

Fig. 7 Disturbances' estimation performance with the $\mathrm{C} 1$ controller

\section{Working condition 2: high-frequency motion}

To further indicate the high-performance tracking effects of the synthesized $\mathrm{C} 1$ controller, we employ the desired motion trajectory $\chi_{1 d}(t)=10 \sin (6 \pi t) \mathrm{mm}$ which is displayed in Fig. 10. Moreover, we can see from Fig. 10 that the output $\chi_{1}$ under the $\mathrm{C} 1$ controller can track $\chi_{1 d}$ closely. Furthermore, the tracking errors and performance indices of the employed five controllers are shown from Figs. 11, 12, 13. All results indicate the high tracking performance of $\mathrm{C} 1$. In addition, from Fig. 14, we can make out that the actual control input is always within the preset ranges and presents corresponding rules, even in the case of input saturation.

Fig. 8 Estimation performance of nonlinear functions with the $\mathrm{C} 1$ controller

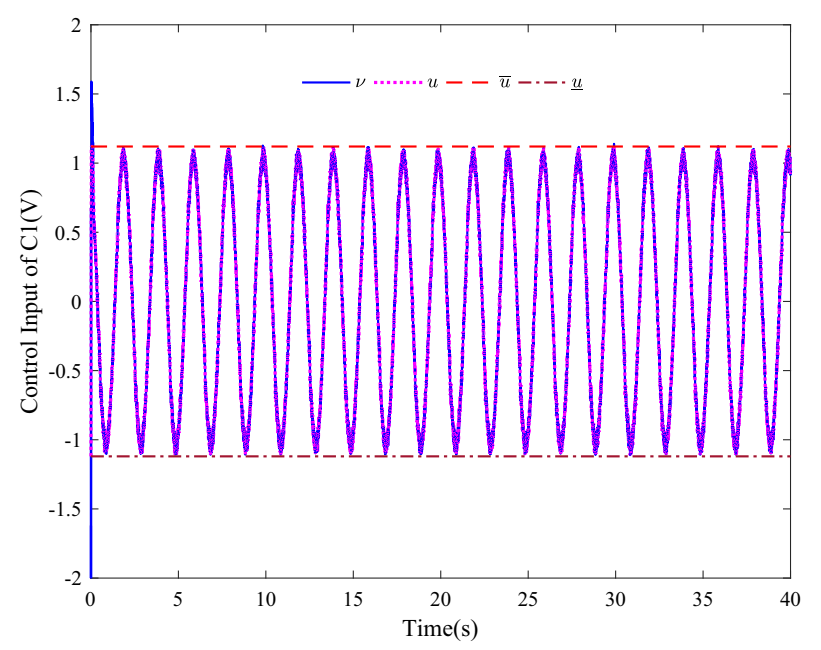

Fig. 9 Control input $u$ with its upper and lower bounds of the $\mathrm{C} 1$ controller

\section{Conclusion}

In this article, a ESO-based NN adaptive motion control strategy has been proposed for a family of servomechanisms with input saturation, which can be adapted to the working condition where some state information can be measured. By comparing several controllers, the compensation performances for disturbance, nonlinear dynamics, and input saturation have been verified. Especially, the estimationerror-and-tracking-error-based NN adaptive control has good approximation ability to function uncertainties including the nonsmooth unknown nonlinear dynamics. In addition, even when the system is saturated, the actual control input still does not exceed the preset limits. Moreover, the system shows 


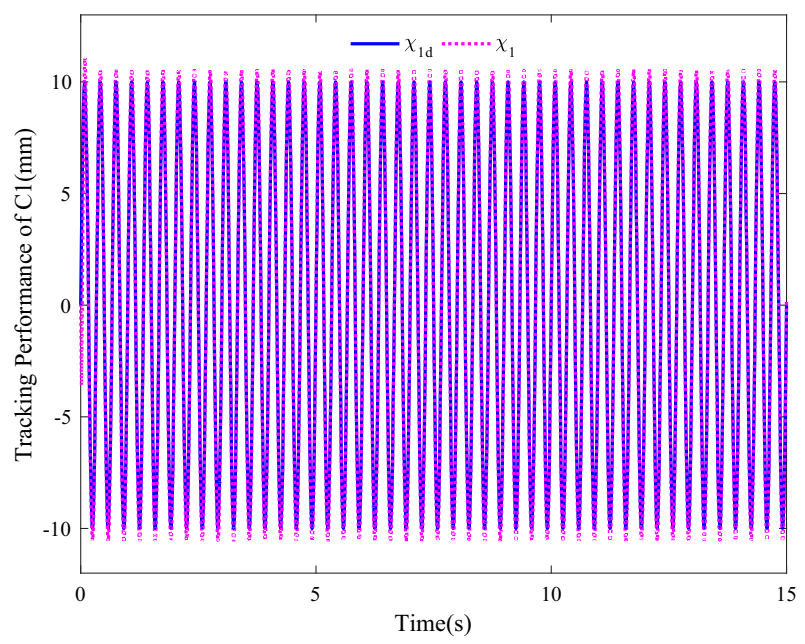

Fig. 10 The tracking performance of $\mathrm{C} 1$
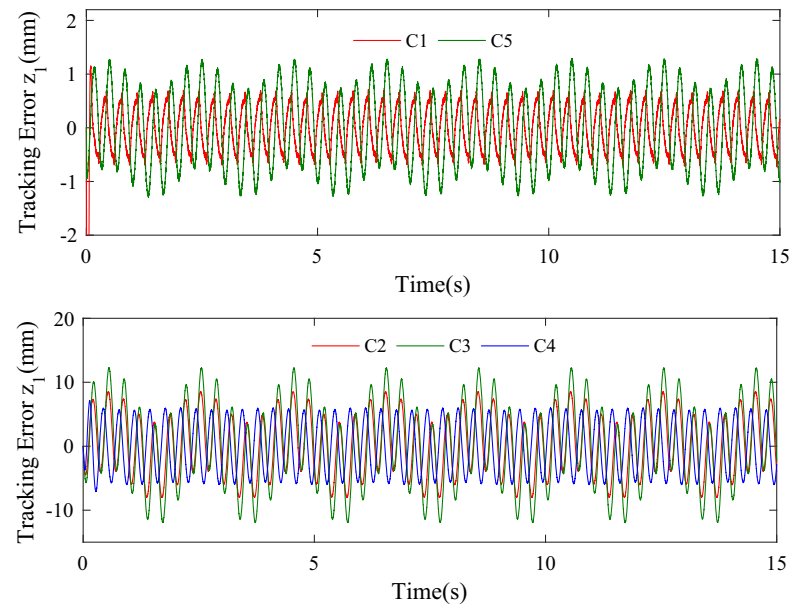

Fig. 11 Tracking errors of the contradistinctive controllers

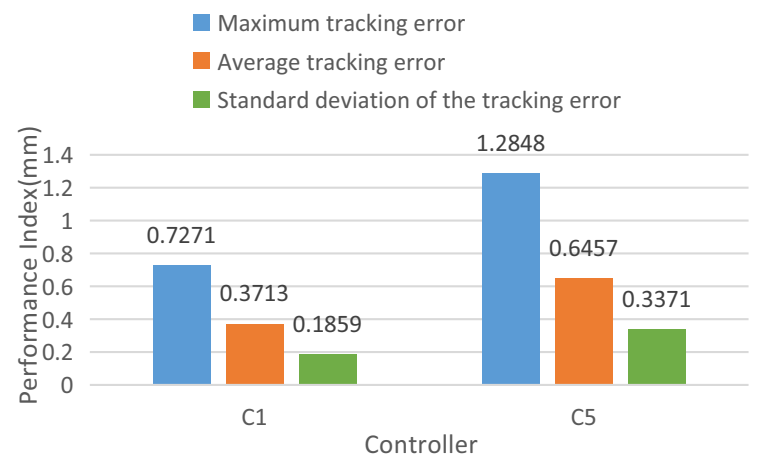

Fig. 12 Performance indices of $\mathrm{C} 1$ and $\mathrm{C} 5$ controllers during last $1 \mathrm{~s}$

high-performance tracking effects with the joint action of ESO and NN. Finally, strict theoretical proof has been presented to ensure the stability of the synthesized closed-loop controller.

Acknowledgements We are grateful to the reviewers for sparing their time and efforts to manage this manuscript.

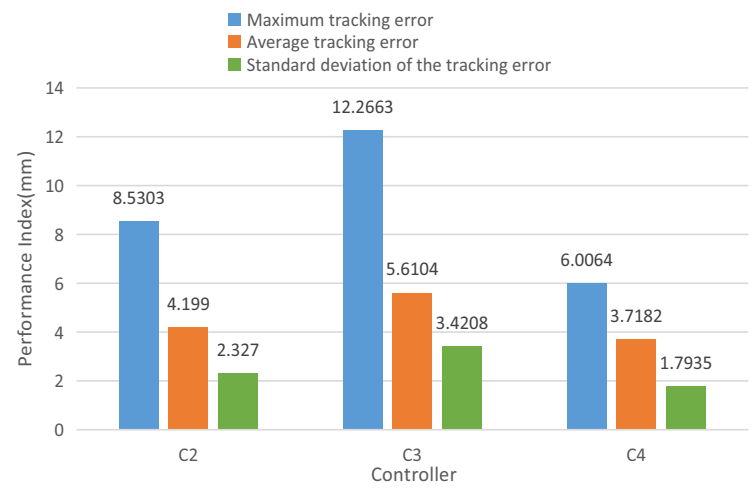

Fig. 13 Performance indices of $\mathrm{C} 2, \mathrm{C} 3$, and $\mathrm{C} 4$ controllers during last $1 \mathrm{~s}$

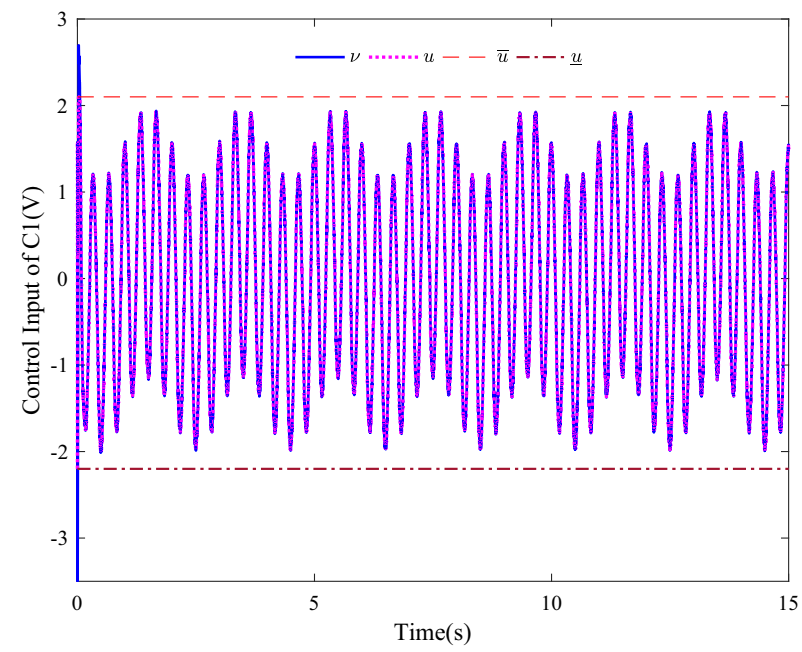

Fig. 14 Control input $u$ with its upper and lower bounds of the $\mathrm{C} 1$

\section{Declarations}

Conflict of interest On behalf of all authors, the corresponding author states that there is no conflict of interest. This work was supported in part by the National Natural Science Foundation of China under Grant 52005249 , in part by the Natural Science Foundation of the Jiangsu Higher Education Institutions of China under Grant 20KJB460019 and in part by the Key Research \& Development Program of Jiangsu Province under Grant BE2019007-3.

Open Access This article is licensed under a Creative Commons Attribution 4.0 International License, which permits use, sharing, adaptation, distribution and reproduction in any medium or format, as long as you give appropriate credit to the original author(s) and the source, provide a link to the Creative Commons licence, and indicate if changes were made. The images or other third party material in this article are included in the article's Creative Commons licence, unless indicated otherwise in a credit line to the material. If material is not included in the article's Creative Commons licence and your intended use is not permitted by statutory regulation or exceeds the permitted use, you will need to obtain permission directly from the copyright holder. To view a copy of this licence, visit http://creativecomm ons.org/licenses/by/4.0/. 


\section{Appendix A}

Proof of Theorem 1 Consider a non-negative Lyapunov function candidate $V_{L}$ which consists of state estimation errors, disturbance estimation errors, NNs weights approximate errors, tracking errors, and compensating signals as follows:

$$
\begin{aligned}
V_{L}= & \frac{1}{2} e_{1}^{2}+\frac{1}{2} e_{2}^{2}+\frac{1}{2} e_{3}^{2}+\frac{1}{2} \phi_{1}^{2}+\frac{1}{2} \phi_{2}^{2}+\frac{1}{2} \phi_{3}^{2}+\frac{1}{2} \zeta^{T} E_{o} \zeta \\
& +\frac{1}{2} \delta^{T} Y_{o} \delta+\frac{1}{2} \tilde{W}_{2}^{T} \Upsilon_{2}^{-1} \tilde{W}_{2}+\frac{1}{2} \tilde{W}_{3}^{T} \Upsilon_{3}^{-1} \tilde{W}_{3} \\
& +\frac{1}{2} \tilde{G}^{T} \Gamma^{-1} \tilde{G}
\end{aligned}
$$

After differentiating both sides of (A.1) with time and substituting (18), (19), (24), (27), (32), (35) into it, one obtains

$$
\begin{aligned}
\dot{V}_{L}= & e_{1}\left(-k_{1} e_{1}+e_{2}\right)+e_{2}\left[e_{3}-k_{2} e_{2}+k_{2} \tilde{\chi}_{2}+\tilde{W}_{2}^{T} \eta_{2}\left(\bar{\chi}_{2 d}\right)\right] \\
& +e_{2}\left(-e_{1}+\tilde{\chi}_{D 2}+\tilde{N}_{2}-k_{1}^{2} e_{1}+k_{1} e_{2}-k_{1}^{2} \phi_{1}+k_{1} \phi_{2}\right) \\
& +e_{3}\left[-k_{3} e_{3}+\tilde{W}_{3}^{T} \eta_{3}\left(\bar{\chi}_{3 d}\right)+\tilde{N}_{3}+\tilde{G}^{T} \psi\left(\chi_{3}\right)+\mu\left(\chi_{3}\right)+\tilde{\chi}_{D 3}\right] \\
& -e_{2} e_{3}+\phi_{1}\left(-k_{1} \phi_{1}+\phi_{2}\right)+\phi_{2}\left[-k_{2} \phi_{2}+\phi_{3}+\left(v_{2, c}-v_{2}\right)\right] \\
& -\frac{1}{2} \omega_{o 1}\|\zeta\|^{2}+\zeta^{T} E_{o}\left[H_{1} \frac{\tilde{W}_{2}^{T} \eta_{2}\left(\bar{\chi}_{2 d}\right)+\tilde{N}_{2}}{\omega_{o 1}}+H_{2} \frac{\alpha_{1}(t)}{\omega_{o 1}^{2}}\right] \\
& +\phi_{3}\left(-k_{3} \phi_{3}+\Delta u\right)-\frac{1}{2} \omega_{o 2}\|\delta\|^{2}+\delta^{T} Y_{o} S_{1}\left[\tilde{W}_{3}^{T} \eta_{3}\left(\bar{\chi}_{3 d}\right)+\tilde{N}_{3}\right] \\
& +\delta^{T} Y_{o} S_{1}\left[\tilde{G}^{T} \psi_{p}\left(\chi_{3}\right)+\mu\left(\chi_{3}\right)\right]+\delta^{T} Y_{o} S_{2} \frac{\alpha_{2}(t)}{\omega_{o 2}} \\
& -\tilde{W}_{2}^{T} \Upsilon_{2}^{-1} \dot{\hat{W}}_{2}-\tilde{W}_{3}^{T} \Upsilon_{3}^{-1} \dot{\hat{W}}_{3}-\tilde{G}^{T} \Gamma^{-1} \dot{\hat{G}} .
\end{aligned}
$$

Moreover, we can reorganize (A.2) as

$$
\begin{aligned}
\dot{V}_{L}= & -k_{1} e_{1}^{2}-\left(k_{2}-k_{1}\right) e_{2}^{2}-k_{3} e_{3}^{2}-k_{1}^{2} e_{1} e_{2} \\
& +k_{2} e_{2} \tilde{\chi}_{2}+e_{2} \tilde{\chi}_{D 2}+e_{2} \tilde{N}_{2}+e_{3} \tilde{N}_{3}+\mu\left(\chi_{3}\right) e_{3}+e_{3} \tilde{\chi}_{D 3} \\
& -k_{1}^{2} e_{2} \phi_{1}+k_{1} e_{2} \phi_{2}-k_{1} \phi_{1}^{2}+\phi_{1} \phi_{2}-k_{2} \phi_{2}^{2}+\phi_{2} \phi_{3} \\
& +\phi_{2}\left(v_{2, c}-v_{2}\right)-k_{3} \phi_{3}^{2}+\phi_{3} \Delta u-\frac{1}{2} \omega_{o 1}\|\zeta\|^{2}+\frac{1}{\omega_{o 1}} \zeta^{T} E_{o} H_{1} \tilde{N}_{2} \\
& +\zeta^{T} E_{o} H_{2} \frac{\alpha_{1}(t)}{\omega_{o 1}^{2}}-\frac{1}{2} \omega_{o 2}\|\delta\|^{2}+\delta^{T} Y_{o} S_{1} \tilde{N}_{3}+\delta^{T} Y_{o} S_{1} \mu\left(\chi_{3}\right) \\
& +\delta^{T} Y_{o} S_{2} \frac{\alpha_{2}(t)}{\omega_{o 2}}+\tilde{W}_{2}^{T} \eta_{2}\left(\bar{\chi}_{2 d}\right) e_{2}+\frac{1}{\omega_{o 1}} \tilde{W}_{2}^{T} \eta_{2}\left(\bar{\chi}_{2 d}\right) \zeta^{T} E_{o} H_{1} \\
& -\tilde{W}_{2}^{T} \Upsilon_{2}^{-1} \dot{\hat{W}}_{2}+\tilde{W}_{3}^{T} \eta_{3}\left(\bar{\chi}_{3 d}\right) e_{3}+\tilde{W}_{3}^{T} \eta_{3}\left(\bar{\chi}_{3 d}\right) \delta^{T} Y_{o} S_{1} \\
& -\tilde{W}_{3}^{T} \Upsilon_{3}^{-1} \hat{\hat{W}}_{3}+\tilde{G}^{T} \psi\left(\chi_{3}\right) e_{3}+\tilde{G}^{T} \psi_{p}\left(\chi_{3}\right) \delta^{T} Y_{o} S_{1}-\tilde{G}^{T} \Gamma^{-1} \dot{\hat{G}} .
\end{aligned}
$$

Noting the definitions of $\zeta$ and $\delta$ as well as (20), (21), (22), we have

$$
\zeta^{T} E_{o} H_{1}=-\frac{1}{2} \zeta_{1}+\zeta_{2}-\frac{1}{2} \zeta_{3}, \delta^{T} Y_{o} S_{1}=\frac{1}{2} \delta_{1}-\frac{1}{2} \delta_{2} .
$$

Based on (36) and (A.4), we can get the upper bound of (A.3) as

$$
\begin{aligned}
& \dot{V}_{L} \leq-k_{1} e_{1}^{2}-\left(k_{2}-k_{1}\right) e_{2}^{2}-k_{3} e_{3}^{2}-k_{1}^{2} e_{1} e_{2} \\
&+k_{2} e_{2} \tilde{\chi}_{2}+e_{2} \tilde{\chi}_{D 2}+e_{2} \tilde{N}_{2}+e_{3} \tilde{N}_{3}+\mu\left(\chi_{3}\right) e_{3}+e_{3} \tilde{\chi}_{D 3} \\
&-k_{1}^{2} e_{2} \phi_{1}+k_{1} e_{2} \phi_{2}-k_{1} \phi_{1}^{2}+\phi_{1} \phi_{2}-k_{2} \phi_{2}^{2}+\phi_{2} \phi_{3}+\rho_{G} \tilde{G}^{T} \hat{G} \\
&+\phi_{2}\left(v_{2, c}-v_{2}\right)-k_{3} \phi_{3}^{2}+\phi_{3} \Delta u-\frac{1}{2} \omega_{o 1}\|\zeta\|^{2}+\frac{1}{\omega_{o 1}} \zeta^{T} E_{o} H_{1} \tilde{N}_{2} \\
&+\zeta^{T} E_{o} H_{2} \frac{\alpha_{1}(t)}{\omega_{o 1}^{2}}-\frac{1}{2} \omega_{o 2}\|\delta\|^{2}+\delta^{T} Y_{o} S_{1} \tilde{N}_{3}+\delta^{T} Y_{o} S_{1} \mu\left(\chi_{3}\right)+ \\
& \delta^{T} Y_{o} S_{2} \frac{\alpha_{2}(t)}{\omega_{o 2}}+\tilde{W}_{2}^{T} \eta_{2}\left(\bar{\chi}_{2 d}\right) \tilde{\chi}_{2}+\rho_{2} \tilde{W}_{2}^{T} \hat{W}_{2}-\frac{1}{2} \tilde{W}_{3}^{T} \eta_{3}\left(\bar{\chi}_{3 d}\right) \delta_{2} \\
& \quad+\frac{1}{\omega_{o 1}} \tilde{W}_{2}^{T} \eta_{2}\left(\bar{\chi}_{2 d}\right)\left(\zeta_{2}-\frac{1}{2} \zeta_{3}\right)+\rho_{3} \tilde{W}_{3}^{T} \hat{W}_{3}-\frac{1}{2} \tilde{G}^{T} \psi_{p}\left(\chi_{3}\right) \delta_{2} .
\end{aligned}
$$

Depending on Young's inequality, one yields

$$
\begin{aligned}
& \tilde{W}_{2}^{T} \hat{W}_{2}=-\left\|\tilde{W}_{2}\right\|^{2}+\tilde{W}_{2}^{T} W_{2} \leq-\frac{1}{2}\left\|\tilde{W}_{2}\right\|^{2}+\frac{1}{2}\left\|W_{2}\right\|^{2} \\
& \tilde{W}_{3}^{T} \hat{W}_{3}=-\left\|\tilde{W}_{3}\right\|^{2}+\tilde{W}_{3}^{T} W_{3} \leq-\frac{1}{2}\left\|\tilde{W}_{3}\right\|^{2}+\frac{1}{2}\left\|W_{3}\right\|^{2} \\
& \tilde{G}^{T} \hat{G}=-\|\tilde{G}\|^{2}+\tilde{G}^{T} G \leq-\frac{1}{2}\|\tilde{G}\|^{2}+\frac{1}{2}\|G\|^{2} .
\end{aligned}
$$

Moreover, we have

$$
\begin{aligned}
& \left|\tilde{N}_{2}\right| \leq p_{2}\left(\left|e_{1}\right|+\left|e_{2}\right|+\left|\phi_{1}\right|+\left|\phi_{2}\right|\right) \\
& \left|\tilde{N}_{3}\right| \leq p_{3}\left(\left|e_{1}\right|+\left|e_{2}\right|+\left|e_{3}\right|+\left|\phi_{1}\right|+\left|\phi_{2}\right|+\left|\phi_{3}\right|+\varepsilon_{N 3}\right) \\
& \left|\mu\left(\chi_{3}\right)\right| \leq \mu_{M},\left|v_{2, c}-v_{2}\right| \leq \varepsilon_{f},|\Delta u| \leq \varepsilon_{s} \\
& \left|\alpha_{1}(t)\right| \leq \alpha_{1 M},\left|\alpha_{2}(t)\right| \leq \alpha_{2 M},
\end{aligned}
$$

where $p_{2}, p_{3}, \varepsilon_{N 3}, \mu_{M}, \varepsilon_{f}, \varepsilon_{s}, \alpha_{1 M}$, and $\alpha_{2 M}$ indicate some positive constants.

Based on (A.6) and (A.7), we can rearrange (A.5) as

$\dot{V}_{L} \leq-k_{1} e_{1}^{2}-\left(k_{2}-k_{1}-p_{2}\right) e_{2}^{2}-\left(k_{3}-p_{3}\right) e_{3}^{2}-k_{1} \phi_{1}^{2}-k_{2} \phi_{2}^{2}$

$-k_{3} \phi_{3}^{2}+\left|\phi_{1}\right|\left|\phi_{2}\right|+\left|\phi_{2}\right|\left|\phi_{3}\right|+\varepsilon_{f}\left|\phi_{2}\right|+\varepsilon_{s}\left|\phi_{3}\right|+p_{3}\left|e_{1}\right|\left|e_{3}\right|$

$+p_{2}\left|e_{2}\right|\left(\left|\phi_{1}\right|+\left|\phi_{2}\right|\right)+p_{3}\left|e_{3}\right|\left(\left|\phi_{1}\right|+\left|\phi_{2}\right|+\left|\phi_{3}\right|+\varepsilon_{N 3}\right)$

$+\left(k_{1}^{2}+p_{2}\right)\left|e_{1}\right|\left|e_{2}\right|+p_{3}\left|e_{2}\right|\left|e_{3}\right|+k_{2}\left|e_{2}\right|\left|\tilde{\chi}_{2}\right|+\left|e_{2}\right|\left|\tilde{\chi}_{D 2}\right|$

$+\mu_{M}\left|e_{3}\right|+\left|e_{3}\right|\left|\tilde{\chi}_{D 3}\right|-\frac{1}{2} \omega_{o 1}\|\zeta\|^{2}+\frac{p_{2}}{\omega_{o 1}}\|\zeta\|\left\|E_{o} H_{1}\right\|\left(\left|e_{1}\right|+\left|e_{2}\right|\right)$

$+\frac{p_{2}}{\omega_{o 1}}\|\zeta\|\left\|E_{o} H_{1}\right\|\left(\left|\phi_{1}\right|+\left|\phi_{2}\right|\right)+\|\zeta\|\left\|E_{o} H_{2}\right\| \frac{\alpha_{1 M}}{\omega_{o 1}^{2}}-\frac{1}{2} \omega_{o 2}\|\delta\|^{2}$

$+p_{3}\|\delta\|\left\|Y_{o} S_{1}\right\|\left(\left|e_{1}\right|+\left|e_{2}\right|+\left|e_{3}\right|+\left|\phi_{1}\right|+\left|\phi_{2}\right|+\left|\phi_{3}\right|+\varepsilon_{N 3}\right)$

$+\mu_{M}\|\delta\|\left\|Y_{o} S_{1}\right\|+\|\delta\|\left\|Y_{o} S_{2}\right\| \frac{\alpha_{2 M}}{\omega_{o 2}}+\left\|\tilde{W}_{2}\right\|\left\|\eta_{2}\left(\bar{\chi}_{2 d}\right)\right\|\left|\tilde{\chi}_{2}\right|$

$+\frac{3}{2 \omega_{01}}\left\|\tilde{W}_{2}\right\|\left\|\eta_{2}\left(\bar{\chi}_{2 d}\right)\right\|\|\zeta\|+\frac{1}{2}\left\|\tilde{W}_{3}\right\|\left\|\eta_{3}\left(\bar{\chi}_{3 d}\right)\right\|\|\delta\|$

$-\frac{1}{2} \rho_{2}\left\|\tilde{W}_{2}\right\|^{2}-\frac{1}{2} \rho_{3}\left\|\tilde{W}_{3}\right\|^{2}+\frac{1}{2}\|\tilde{G}\|\left\|\psi\left(\chi_{3}\right)\right\|\|\delta\|$

$-\frac{1}{2} \rho_{G}\|\tilde{G}\|^{2}+\frac{1}{2} \rho_{2}\left\|W_{2}\right\|^{2}+\frac{1}{2} \rho_{3}\left\|W_{3}\right\|^{2}+\frac{1}{2} \rho_{G}\|G\|^{2}$. 
Applying on Young's inequality, thus one gets

$$
\begin{aligned}
\dot{V}_{L} \leq & -k_{e 1} e_{1}^{2}-k_{e 2} e_{2}^{2}-k_{e 3} e_{3}^{2}-k_{\phi 1} \phi_{1}^{2}-k_{\phi 2} \phi_{2}^{2} \\
& -k_{\phi 3} \phi_{3}^{2}-k_{\zeta}\|\zeta\|^{2}-k_{\delta}\|\delta\|^{2}-k_{\tilde{W}_{2}}\left\|\tilde{W}_{2}\right\|^{2} \\
& -k_{\tilde{W}_{3}}\left\|\tilde{W}_{3}\right\|^{2}-k_{\tilde{G}}\|\tilde{G}\|^{2}+\varepsilon_{L},
\end{aligned}
$$

where

$$
\begin{aligned}
k_{e 1}= & k_{1}-q_{1}-\frac{1}{2} p_{3}-\frac{p_{2}^{2}}{2}-\frac{p_{3}^{2}}{2}, \\
k_{e 2}= & k_{2}-k_{1}-2 p_{2}-\frac{1}{2}\left(p_{3}+p_{2}^{2}+p_{3}^{2}\right) \\
& -\frac{\left(k_{1}^{2}+p_{2}\right)^{2}}{q_{1}}-\frac{k_{2}^{2}}{q_{2}}-q_{3}-q_{4}, \\
k_{e 3}= & k_{3}-\frac{7}{2} p_{3}-\frac{p_{3}^{2}}{2}-\frac{1}{2}, \\
k_{\phi 1}= & k_{1}-\frac{1}{2}-\frac{1}{2} p_{2}-\frac{1}{2} p_{3}-\frac{p_{2}^{2}}{2}-\frac{p_{3}^{2}}{2}, \\
k_{\phi 2}= & k_{2}-\frac{3}{2}-\frac{1}{2} p_{2}-\frac{1}{2} p_{3}-\frac{p_{2}^{2}}{2}-\frac{p_{3}^{2}}{2}, \\
k_{\phi 3}= & k_{3}-1-\frac{1}{2} p_{3}-\frac{p_{3}^{2}}{2}, \\
k_{\zeta}= & \frac{1}{2} \omega_{o 1}-q_{2} \omega_{o 1}^{2}-\frac{2\left\|E_{o} H_{1}\right\|^{2}}{\omega_{o 1}^{2}}-\frac{\omega_{o 1}^{4}}{q_{3}} \\
& -\frac{\left\|E_{o} H_{2}\right\|^{2}}{2 \omega_{o 1}^{4}}-q_{5}-\frac{1}{2} \\
& +\alpha_{1 M}^{2}+\frac{1}{2} \mu_{M}^{2}+\frac{1}{2} \varepsilon_{f}^{2}+\frac{1}{2} \varepsilon_{s}^{2}+\varepsilon_{N 3}^{2} p_{3}^{2}, \\
k_{\delta}= & \frac{1}{2} \omega_{o 2}-\frac{\omega_{o 2}^{2}}{q_{4}}-4\left\|Y_{o} S_{1}\right\|^{2}-\frac{1}{2 \omega_{o 2}^{2}}\left\|Y_{o} S_{2}\right\|^{2}-1, \\
k_{\tilde{W}_{2}}= & \frac{1}{2} \rho_{2}-\frac{\omega_{o 1}^{2} \eta_{2 M}^{2}}{q_{5}}-\frac{9 \eta_{2 M}^{2}}{8 \omega_{o 1}^{2}}, k_{\tilde{W}_{3}}=\frac{1}{2} \rho_{3}-\frac{\eta_{3 M}^{2}}{8}, \\
k_{\tilde{G}}= & \frac{1}{2} \rho_{G}-\frac{\psi_{M}^{2}}{8}, \\
\varepsilon_{L}= & \frac{1}{2} \rho_{2}\left\|W_{2}\right\|^{2}+\frac{1}{2} \rho_{3}\left\|W_{3}\right\|^{2}+\frac{1}{2} \rho_{G}\|G\|^{2} \\
&
\end{aligned}
$$

in which $q_{1}, q_{2}, q_{3}, q_{4}, \eta_{2 M}, \eta_{3 M}$, and $\psi_{M}$ indicate some positive constants. Specially, $\eta_{2 M}, \eta_{3 M}$, and $\psi_{M}$ denote the upper bounds of $\eta_{2}\left(\bar{\chi}_{2 d}\right), \eta_{3}\left(\bar{\chi}_{3 d}\right)$, and $\psi\left(\chi_{3}\right)$, respectively.

From (A.9), we have

$\dot{V}_{L} \leq-\rho_{L} V_{L}+\varepsilon_{L}$,

where $\rho_{L}=2 \min \left\{k_{e 1}, k_{e 2}, k_{e 3}, k_{\phi 1}, k_{\phi 2}, k_{\phi 3}, k_{\zeta} / \lambda_{\max }\left(E_{o}\right)\right.$, $k_{\delta} / \lambda_{\max }\left(Y_{o}\right), k_{\tilde{W}_{2}} / \lambda_{\max }\left(\Upsilon_{2}^{-1}\right), k_{\tilde{W}_{3}} / \lambda_{\max }\left(\Upsilon_{3}^{-1}\right), k_{\tilde{G}} /$ $\left.\lambda_{\max }\left(\Gamma^{-1}\right)\right\}$ indicates a positive constant.
Furthermore, we have

$V_{L}(t) \leq V_{L}(0) e^{-\rho_{L} t}+\frac{\varepsilon_{L}}{\rho_{L}}\left(1-e^{-\rho_{L} t}\right)$.

Noting the expressions in (A.1) and (A.12), we can infer that all system signals in the considered closed-loop servomechanism always stay bounded [40]. Consequently, all the results in Theorem 1 have been completely proved.

\section{References}

1. Liu H, Li S (2012) Speed control for PMSM servo system using predictive functional control and extended state observer. IEEE Trans Ind Electron 59(2):1171-1183

2. Yang $G$, Zhu $T$, Wang $H$, Yang $F$ (2021) Disturbancecompensation-based multilayer neuroadaptive control of MIMO uncertain nonlinear systems. IEEE Trans Circuits Syst II Express Briefs. https://doi.org/10.1109/TCSII.2021.3116872

3. Sun X, Chen L, Jiang H, Yang Z, Chen J, Zhang W (2016) High-performance control for a bearingless permanent-magnet synchronous motor using neural network inverse scheme plus internal model controllers. IEEE Trans Ind Electron 63(6):3479-3488

4. Wang X, Wang W, Li L, Shi J, Xie B (2019) Adaptive control of dc motor servo system with application to vehicle active steering. IEEE/ASME Trans Mechatron 24(3):1054-1063

5. Zheng D, Xu H (2016) Adaptive backstepping-flatness control based on an adaptive state observer for a torque tracking electrohydraulic system. IEEE/ASME Trans Mechatron 21(5):2440-2452

6. Yang X, Zheng X, Chen Y (2017) Position tracking control law for an electro-hydraulic servo system based on backstepping and extended differentiator. IEEE/ASME Trans Mechatron 23(1):132-140

7. Liu YJ, Zeng Q, Liu L, Tong S (2018) An adaptive neural network controller for active suspension systems with hydraulic actuator. IEEE Trans Syst Man Cybern Syst 50(12):5351-5360

8. Yang G, Yao J (2020) High-precision motion servo control of double-rod electro-hydraulic actuators with exact tracking performance. ISA Trans 103:266-279

9. Sun L, Zheng Z (2017) Disturbance-observer-based robust backstepping attitude stabilization of spacecraft under input saturation and measurement uncertainty. IEEE Trans Ind Electron 64(10):7994-8002

10. Xu B, Wang D, Zhang Y, Shi Z (2017) DOB-based neural control of flexible hypersonic flight vehicle considering wind effects. IEEE Trans Ind Electron 64(11):8676-8685

11. Lu L, Yao B (2014) Online constrained optimization based adaptive robust control of a class of MIMO nonlinear systems with matched uncertainties and input/state constraints. Automatica 50(3):864-873

12. Yang G, Yao J, Le G, Ma D (2016) Adaptive integral robust control of hydraulic systems with asymptotic tracking. Mechatronics 40:78-86

13. Wang S, Na J, Ren X (2018) RISE-based asymptotic prescribed performance tracking control of nonlinear servo mechanisms. IEEE Trans Syst Man Cybern Syst 48(12):2359-2370

14. Yang J, Li S, Yu X (2013) Sliding-mode control for systems with mismatched uncertainties via a disturbance observer. IEEE Trans Ind Electron 60(1):160-169

15. Ding S, Zheng WX (2016) Nonsingular terminal sliding mode control of nonlinear second-order systems with input saturation. Int J Robust Nonlinear Control 26(9):1857-1872 
16. Won D, Kim W, Tomizuka M (2017) High-gain-observerbased integral sliding mode control for position tracking of electrohydraulic servo systems. IEEE/ASME Trans Mechatron 22(6):2695-2704

17. Liu C, Luo G, Duan X, Chen Z, Zhang Z, Qiu C (2019) Adaptive LADRC-based disturbance rejection method for electromechanical servo system. IEEE Trans Ind Appl 56(1):876-889

18. Yang G, Yao J (2019) Output feedback control of electro-hydraulic servo actuators with matched and mismatched disturbances rejection. J Frankl Inst 356(16):9152-9179

19. Yu J, Shi P, Dong W, Chen B, Lin C (2015) Neural networkbased adaptive dynamic surface control for permanent magnet synchronous motors. IEEE Trans Neural Netw 26(3):640-645

20. Pan Y, Liu Y, Xu B, Yu H (2016) Hybrid feedback feedforward: an efficient design of adaptive neural network control. Neural Netw $76: 122-134$

21. Wang Z, Hu C, Zhu Y, He S, Yang K, Zhang M (2017) Neural network learning adaptive robust control of an industrial linear motor-driven stage with disturbance rejection ability. IEEE Trans Ind Inf 13(5):2172-2183

22. Yang G, Yao J, Ullah N (2021) Neuroadaptive control of saturated nonlinear systems with disturbance compensation. ISA Trans. https://doi.org/10.1016/j.isatra.2021.04.017

23. Liu YJ, Zeng Q, Tong S, Chen CP, Liu L (2019) Adaptive neural network control for active suspension systems with time-varying vertical displacement and speed constraints. IEEE Trans Ind Electron 66(12):9458-9466

24. Wang S, Yu H, Yu J, Na J, Ren X (2020) Neural-network-based adaptive funnel control for servo mechanisms with unknown deadzone”. IEEE Trans Syst Man Cybern Syst 50(4):1383-1394

25. Ramirez A, Garrido R, Mondie S (2015) Velocity control of servo systems using an integral retarded algorithm. ISA Trans 58:357-366

26. Zhang Y, Yan P, Zhang Z (2016) High precision tracking control of a servo gantry with dynamic friction compensation. ISA Trans 62:349-356

27. Zhao L, Xia Y, Yang Y, Liu Z (2017) Multicontroller positioning strategy for a pneumatic servo system via pressure feedback. IEEE Trans Ind Electron 64(6):4800-4809

28. Elsousy FFM (2013) Adaptive dynamic sliding-mode control system using recurrent RBFN for high-performance induction motor servo drive. IEEE Trans Ind Inf 9(4):1922-1936
29. Du J, Hu X, Krstić M, Sun Y (2016) Robust dynamic positioning of ships with disturbances under input saturation. Automatica 73(73):207-214

30. Yang Y, Tan J, Yue D (2020) Prescribed performance tracking control of a class of uncertain pure-feedback nonlinear systems with input saturation. IEEE Trans Syst Man Cybern Syst 50(5):1733-1745

31. Yu J, Zhao L, Yu H, Lin C, Dong W (2018) Fuzzy finite-time command filtered control of nonlinear systems with input saturation. IEEE Trans Syst Man Cybern Syst 48(8):2378-2387

32. Li Y, Qiang S, Zhuang X, Kaynak O (2004) Robust and adaptive backstepping control for nonlinear systems using RBF neural networks. IEEE Trans Neural Netw 15(3):693-701

33. Han J (2009) From PID to active disturbance rejection control. IEEE Trans Ind Electron 56(3):900-906

34. Yang G, Wang H, Chen J (2021) Disturbance compensation based asymptotic tracking control for nonlinear systems with mismatched modeling uncertainties. Int J Robust Nonlinear Control 31(8):2993-3010

35. Selmic RR, Lewis FL (2002) Neural-network approximation of piecewise continuous functions: application to friction compensation. IEEE Trans Neural Netw 13(3):745-751

36. Gao Z (2003) Scaling and bandwidth-parameterization based controller tuning. In: Proceedings of American Control Conference, pp 4989-4996

37. Zheng Q, Gao LQ, Gao Z (2012) On validation of extended state observer through analysis and experimentation. J Dyn Syst Meas Contr 134(2):024505

38. Farrell JA, Polycarpou MM, Sharma M, Dong W (2009) Command filtered backstepping. IEEE Trans Autom Control 54(6):1391-1395

39. Dong W, Farrell JA, Polycarpou MM, Djapic V, Sharma M (2012) Command filtered adaptive backstepping. IEEE Trans Control Syst Technol 20(3):566-580

40. Krstic M, Kanellakopoulos I, Kokotovic PV (1995) Nonlinear and adaptive control design. Wiley, New York

Publisher's Note Springer Nature remains neutral with regard to jurisdictional claims in published maps and institutional affiliations. 